\title{
Chemical Mapping at the Atomic Level using Energy Dispersive X-ray Spectroscopy
}

\author{
D. Klenov, ${ }^{*}$ B. Freitag,* H.S. von Harrach, ${ }^{*}$, A.J. D’Alfonso, ${ }^{* *}$ and L.J. Allen** \\ *FEI Company, Building AAE, Achtseweg Noord 5, Eindhoven, The Netherlands \\ **School of Physics, University of Melbourne, Parkville, Victoria 3010, Australia
}

We demonstrate chemical mapping at the atomic level using energy dispersive $\mathrm{x}$-ray spectroscopy (EDS) in $C s$-corrected scanning transmission electron microscopy (STEM). The combination of the increase in current in an atomic sized probe by $C s$-correction and the increase in sensitivity of the Super-X detector [1] allows acquisition of such results within minutes and at high sampling rates. The high speed of the software and excellent $\mathrm{S} / \mathrm{N}$ ratio of the EDS detector enables ultra fast mapping at $>10.000$ spectra per second and minimizes artifacts like sample drift and beam damage.

To benchmark the technology we show results at the atomic level using different materials like perovskites and semiconductors. For the first time atomic chemical information on interfaces is obtained using EDS spectroscopy (figure 1). The sampling rate, field of view $(200 \times 200$ pixels $=5 \times 5 \mathrm{~nm})$ and signal strength presented is much higher when compared to previously reported results using conventional EDS detectors [2],[3]. The dependence of performance on experimental parameters and the absorption edges ( $L$ and $K$ edges) on different elements is discussed.

Theoretical Bloch wave simulations of a PTO-STO interface are shown in figure 2. The simulations for EDS mapping assume that the cross section for x-ray emission (when filling a $K$ or $L$ shell hole) is proportional to the total cross section for $K$ or $L$ shell ionization for a detector which samples the full solid angle and all possible energy losses above the ionization threshold. This ensures that the ionization interaction is as localized as possible and that, like high- angle annular dark field (HAADF) imaging, EDS is an incoherent form of imaging and directly interpretable. The simulations in figure 2 for an interface with no diffusion across the boundary do not exhibit the same intermixing of the $\mathrm{Sr}$ signal at the $\mathrm{Pb}-\mathrm{Sr}$ interface seen in experiment (figure 1). Assuming that the EDS imaging is directly interpretable, this indicates that there are both $\mathrm{Pb}$ and $\mathrm{Sr}$ atoms in the interface. Further simulations are currently underway investigating this.

Finally, as the number of elements in the periodic table accessible for chemical mapping by EDS is much higher than for EELS, EDS mapping provides chemical information at the atomic level, on complex multiphase materials, which was not accessible via EELS in the past.

\section{References}

[1] H.S. von Harrach et al., Microsc. Microanal. 15 (Suppl.2) (2009) 208.

[2] A. J. D’Alfonso, B. Freitag, D. Klenov and L. J. Allen, Phys. Rev. B. 81, 100101R (2010)

[3] M.-W. Chu, S. C. Liou, C.-P. Chang, F.-S. Choa and C. H. Chen, Phys. Rev. Lett. 104, $196101(2010)$ 

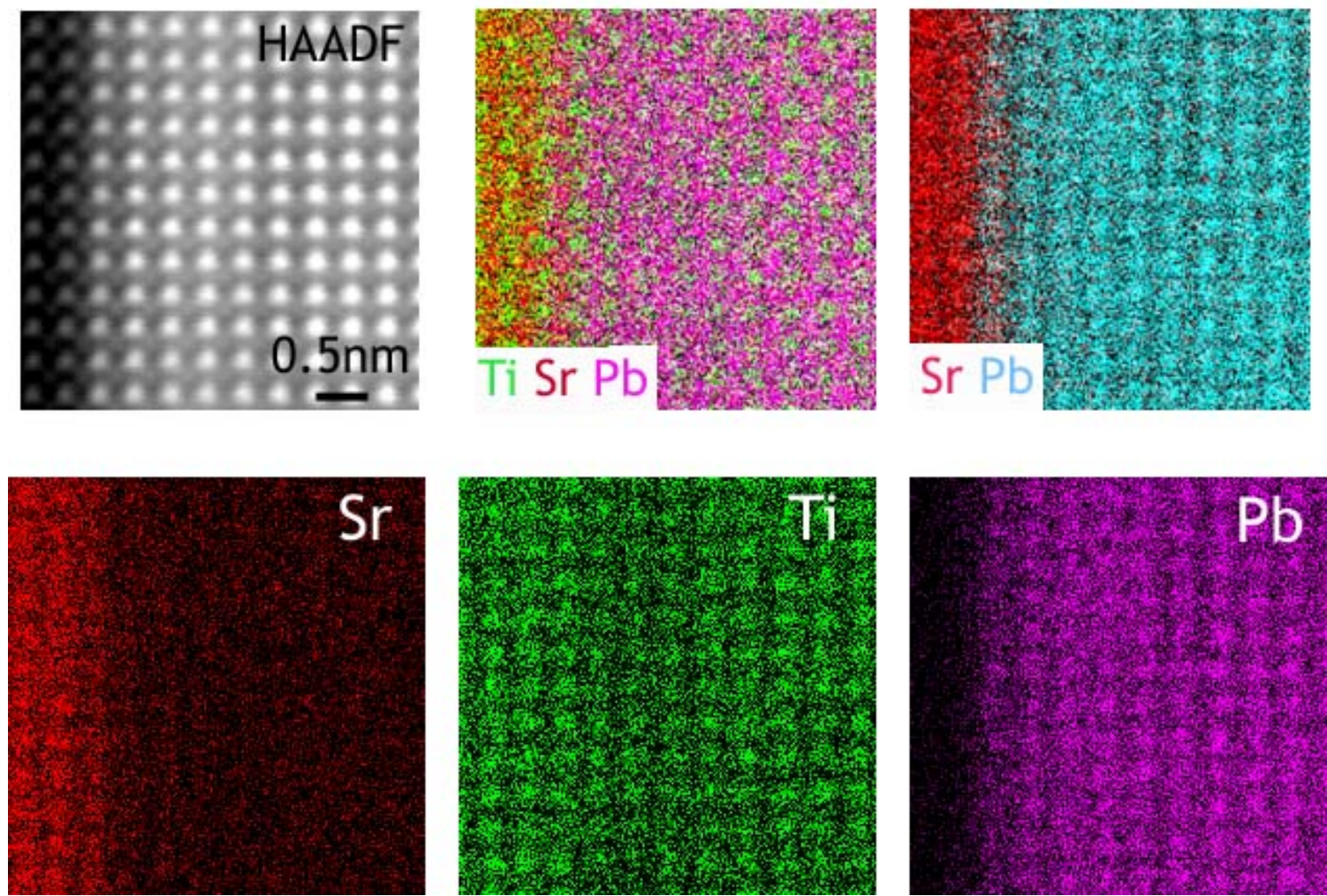

Figure 1 : Atomic resolution EDS map of $\mathrm{SrTiO}_{3} / \mathrm{PbTiO}_{3}$ interface at $200 \mathrm{kV}$ accelerating voltage. The upper left portion shows the HAADF STEM image of the interface and composite images of the $\mathrm{Ti}, \mathrm{Pb}, \mathrm{Sr}$ and the $\mathrm{Sr}, \mathrm{Pb}$ signals. In the lower part the maps of the individual elements are shown. The data presented is raw data obtained by only extracting the intensities of the characteristic absorption edge of the elements. The mapping size is $200 \times 200$ pixels with a total mapping time of $\sim 300 \mathrm{~s}$. Clearly the positions of the $\mathrm{Sr}, \mathrm{Ti}$ and $\mathrm{Pb}$ atomic columns can be visualized in the $\mathrm{x}$-ray maps revealing the chemical change at the interface.
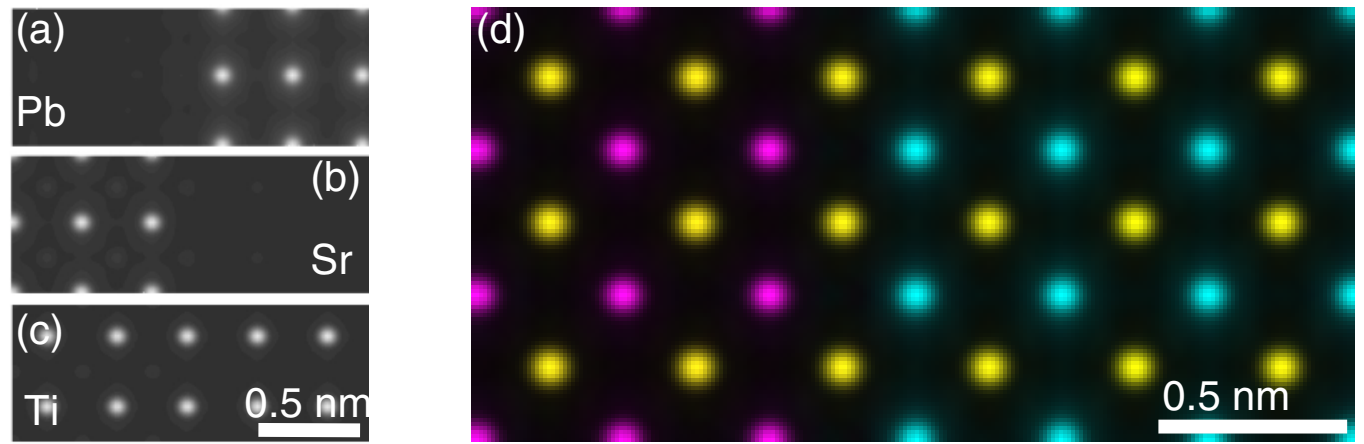

Figure 2: Theoretical simulation of atomic resolution EDS maps of a $\mathrm{SrTiO}_{3} / \mathrm{PbTiO}_{3}$ interface for the (a) $\mathrm{Pb} L_{2,3}$ (b) $\mathrm{Sr} K$ and (c) Ti $K$ edges. The $200 \mathrm{keV}$ probe was assumed to be aberration free with a convergence semi-angle of $21.5 \mathrm{mrad}$. The specimen was assumed to be $50 \mathrm{~nm}$ thick. In (d) we show a color composite map constructed from the $\mathrm{Pb} L_{2,3}$ signal (cyan), the $\mathrm{Sr} K$ signal (magenta) and the Ti K signal (yellow). 